\title{
Effect of the topical application of calcipotriol on the expression levels of zinc finger protein A20 and nuclear factor- $\kappa B$ in the skin lesions of patients with psoriasis vulgaris
}

\author{
YA-JUAN TANG, RU-ZHI ZHANG, XIAO-MING LIU, CHUN-XING XU, SAI CHENG and QI LIU
}

Department of Dermatology, The Third Affiliated Hospital of Soochow University, Changzhou, Jiangsu 213003, P.R. China

Received June 26, 2014; Accepted March 16, 2015

DOI: $10.3892 /$ etm.2015.2887

\begin{abstract}
The aim of the present study was to explore the effect of the topical application of calcipotriol on the expression levels of zinc finger protein A20 and nuclear factor- $\kappa \mathrm{B}$ $(\mathrm{NF}-\kappa \mathrm{B})$ in the skin lesions of patients with psoriasis vulgaris. The calcipotriol ointment was topically applied twice a day for 6 weeks by 26 patients with psoriasis vulgaris. At the end of weeks 2, 4 and 6 after the first application of calcipotriol ointment, the clinical efficacy and Psoriasis Area and Severity Index (PASI) score were compared with those prior to treatment. The expression of zinc finger protein A20 and NF- $\kappa$ B in the skin lesions prior to and following treatment with calcipotriol was measured by immunohistochemical staining and western blotting. At the end of week 6 , the clinical effectiveness rate of calcipotriol was higher compared with that at the end of weeks 2 and $4\left(\chi^{2}=8.12\right.$ and 9.06, respectively; $\left.\mathrm{P}<0.05\right)$. The PASI score declined significantly at the end of weeks 2,4 and $6(\mathrm{t}=9.37,10.54$ and $12.43 ; \mathrm{P}<0.05,0.05$ and 0.001 , respectively). At the end of week 6 , the expression levels of zinc finger protein $\mathrm{A} 20$ and $\mathrm{NF}-\kappa \mathrm{B}$ were significantly lower compared with those prior to treatment $\left(\chi^{2}=3.65\right.$ and 4.17 , respectively; $\mathrm{P}<0.01)$. The expression levels of the two proteins were higher in the skin lesions of patients with psoriasis vulgaris prior to the initiation of treatment than in the skin of a normal control group. Following the 6-week treatment with calcipotriol, the expression levels of the two proteins in the psoriasis skin lesions were significantly lower than they were prior to treatment $(\mathrm{P}<0.01)$. Thus, the present study found that in addition to the typical pathway of $\mathrm{NF}-\kappa \mathrm{B}$ being targeted in the treatment of psoriasis with calcipotriol, the zinc finger protein A20 may also modulate the inflammatory response of psoriasis.
\end{abstract}

Correspondence to: Dr Xiao-Ming Liu, Department of Dermatology, The Third Affiliated Hospital of Soochow University, 185 Juqian Street, Changzhou, Jiangsu 213003, P.R. China E-mail: xiaomingliux@163.com

Key words: psoriasis, calcipotriol, zinc finger protein A20, nuclear factor- $\mathrm{\kappa} \mathrm{B}$

\section{Introduction}

Psoriasis is a common type of chronic inflammatory dermatosis and a long-lasting autoimmune disease characterized by patches of abnormal skin, that is characterized by skin lesions including red, scaly patches, papules and plaques that usually itch. The exact cause and mechanism of action of psoriasis are unclear. It is not entirely a skin disorder and can have a negative impact on many organ systems. They may vary in severity from small and localized to complete body coverage. There are five main types of psoriasis: plaque, guttate, inverse, pustular, and erythrodermic. A previous study indicated that the levels of the typical inflammatory signal nuclear factor- $\kappa \mathrm{B}$ $(\mathrm{NF}-\kappa \mathrm{B})$ are increased in the lymphocytes of the skin lesions and peripheral blood of patients with psoriasis (1). This suggests that $\mathrm{NF}-\kappa \mathrm{B}$ plays an important role as a regulator in the pathological mechanism of psoriasis (1). The zinc finger protein A20, also termed tumor necrosis factor (TNF)- $\alpha$ induced protein-3 (TNFAIP3), is a negative regulating protein of the NF- $\mathrm{B}$ signaling pathway and inhibits the activity of $\mathrm{NF}-\kappa \mathrm{B}$; the expression of TNFAIP $3 \mathrm{mRNA}$ has been found to be negatively associated with the severity of psoriasis (2). It has been confirmed that the vitamin D3 derivative calcipotriol acts as an effective treatment for psoriasis by inhibiting the $\mathrm{NF}-\kappa \mathrm{B}$ signaling pathway (3). However, the detailed mechanism of this and the association between calcipotriol and zinc finger protein A20 in psoriasis are not clear. Therefore, the aim of the present study was to analyze the changes in the expression levels of zinc finger protein A20 and NF- $\kappa$ B in the skin lesions of patients with psoriasis following topical treatment with calcipotriol in order to investigate the possible mechanism of the zinc finger protein A20 in psoriasis and the effect of treatment with calcipotriol.

\section{Materials and methods}

Materials. Calcipotriol ointment $(0.05 \mathrm{mg} / \mathrm{g})$ was purchased from Bright Future Pharmaceutical Laboratories Ltd. (Hong Kong, China). The rabbit anti-human A20 and NF- $\kappa$ B p65 polyclonal primary antibodies were obtained from Abcam (cat no. ab16502, Cambridge, MA, USA) and the horseradishperoxidase (HRP)-labeled goat anti-rabbit IgG secondary antibody was from Pierce Biotechnology, Inc. (cat no. G-21234, Rockford, IL, USA). The quantitative BCA protein kit and 
electrochemical luminescence (EasyBlot ECL) developing system were also purchased from Pierce Biotechnology, Inc. Protease inhibitor cocktail tablets were obtained from Roche Diagnostics (Indianapolis, IN, USA). The MaxVision ${ }^{\mathrm{TT}}$ immunohistochemical staining kit and DAB staining solution were purchased from Maixin Biotechnology Development Co., Ltd. (Fuzhou, China). The upright Olympus BX53 microscope was from Olympus Corporation (Tokyo, Japan).

Patients. A total of 26 patients with psoriasis vulgaris, including 15 males and 11 females with an age range of 20-58 years (mean, $30.56 \pm 9.78$ years) and a disease history of 3-42 months (mean, 10.32 \pm 4.61 months), were recruited for the present study. All patients had a skin lesion area $<30 \%$ of the total body surface area and had not been treated with a systemic or topical application of calcipotriol or any other anti-psoriasis medication for the previous 4 weeks. Control samples were collected from the normal skin at $0.5 \mathrm{~cm}$ from the edge of the pigmented nevus in 18 patients (11 males and 7 females) undergoing resection of pigmented nevi in the outpatient department of the Third Affiliated Hospital of Suzhou University (Changzhou, China). Erythema, scaling, infiltrating hypertrophy and itch were measured by a clinician. The age, gender and locations of the skin lesions were matched between the patient and control groups. The present study was approved by the Ethics Committee of the Third Affiliated Hospital of Suzhou University and informed consent was obtained from the patients.

Calcipotriol administration procedure. Calcipotriol ointment was topically administered to the patients twice a day for 6 weeks. The Psoriasis Area and Severity Index (PASI) was used to evaluate the severity of the skin lesions (4) by sampling the skin with a skin trephine prior to, during and following the calcipotriol therapy.

Immunohistochemical staining. Following regular dewaxing, the slices were stained using the MaxVision ${ }^{\mathrm{Tw}}$ immunohistochemical staining kit according to the manufacturer's instructions. The primary antibodies used were rabbit anti-human A20 and NF- $\mathrm{KB}$ p65 polyclonal antibodies at a dilution of 1:200 and the samples were incubated at $37^{\circ} \mathrm{C}$ for $12 \mathrm{~h}$. They were washed with phosphate-buffered saline and the slices were subsequently developed in $\mathrm{DAB}$ staining solution followed by hematoxylin counterstaining at $37^{\circ} \mathrm{C}$ in a water bath for $2 \mathrm{~h}$, differentiation with $0.1 \%$ hydrochloric acid, regular dehydration and vitrification, and mounting with neutral balsam. The cytoplasms and nuclei of the stained cells were observed under an Olympus BX53 microscope for semi-quantitative analysis using a combination of scores allocated for the percentage of positive cells and the staining intensity. The staining intensity was defined as follows: 0 , negative; 1 , light yellow; 2 , tan; and 3 , dark brown. The percentage of positive cells was defined as follows: 0 , no positive cells; $1, \leq 25 \% ; 2, \sim 26-50 \% ; 3, \sim 51-75 \%$ and; $4,>75 \%$. The product of staining intensity and percentage of positive cells was used to assess the overall staining result: negative $(-), \leq 1$; weak positive $(+), 2$ or 3 ; middle positive $(++), 4$ or 5 ; strong positive $(+++), \geq 6$ and a result of + to +++ was considered as positive expression $(5,6)$.
Western blot analysis. The expression levels of A20 and NF- $\kappa \mathrm{B}$ proteins were measured with western blot analysis, using an improved version of a previously used technique and glyceraldehyde 3-phosphate dehydrogenase as a control (7). The tissue samples were placed in $200 \mu 1$ lysis buffer containing $0.1 \mathrm{M}$ pH 7.5 Tris-HCl, $1 \%$ NP-40 and $0.01 \%$ SDS, and fully ground in a glass homogenizer following the addition of a protease inhibitor cocktail tablet. The proteins were measured with a quantitative BCA protein kit according to the manufacturer's instructions. The extracted proteins were denatured by heating at $95^{\circ} \mathrm{C}$ for $5 \mathrm{~min}$, cooled on ice and loaded (10 $\left.\mu \mathrm{g} / \mathrm{well}\right)$ for electrophoresis on a $10 \%$ SDS-PAGE gel. They were subsequently transferred to a PVDF membrane ( $25 \mathrm{~V}$ for $1 \mathrm{~h}$ ) that was soaked in 5\% skimmed milk, and incubated in $0.1 \%$ Tris-buffered saline and Tween 20 (TBS-T) blocking buffer (Pierce Biotechnology, Inc.) at $4^{\circ} \mathrm{C}$ overnight. The membrane was incubated in a buffer containing the primary antibodies against A20 and NF- $\mathrm{kB}$ p65 (dilution, 1:1,000) for $1 \mathrm{~h}$ and rinsed with TBS-T buffer 5 times, at 3-5 min each time. A buffer containing goat anti-rabbit IgG labeled with HRP was subsequently added (1:5,000 dilution) and the solution was incubated at room temperature for $1 \mathrm{~h}$. Following rinsing with TBS-T buffer and drying, the protein was measured with the an ECL developing system according to the manufacturer's instructions.

Briefly, the membrane was incubated with ECL developing solution for 10-15 min and then a film was placed on the PVDF membrane in a darkroom. The film was then developed using a developing machine (Bio-RAD ChemiDox XRS, Bio-RAD, Hercules, CA, USA) and the exposure time that created a well-developed film was noted down for further runs of this procedure. The developed film was scanned on a computer and the bands were analysed using BandScan5.0 (http://soft. bio1000.com/show-149.html) (8).

Statistical analysis. All data are expressed as the mean \pm standard deviation and were analyzed with SPSS software, version 13.0 (SPSS, Inc., Chicago, IL, USA). The intergroup means, rates and correlations were compared with the Student's t-test, $\chi^{2}$ test and Spearman rank-order correlation analysis, respectively. $\mathrm{P}<0.05$ was considered to indicate a statistically significant difference.

\section{Results}

Comparison of the clinical effectiveness rate and PASI score. At the end of week 6 of treatment with calcipotriol, the erythema, scaling, infiltrating hypertrophy, itch and area of the skin lesions was markedly improved; the effectiveness rate $(57.69 \%, 15 / 26)$ was significantly higher compared with that at the end of week $4(38.46 \%, 10 / 26)$ and week 2 $\left(19.23 \%, 5 / 26 ; \chi^{2}=8.12\right.$ and 9.06, respectively; $\left.\mathrm{P}<0.05\right)$. Compared with that prior to treatment $(10.92 \pm 1.72)$, the PASI score at the end of week $2(8.86 \pm 2.42)$, week $4(6.57 \pm 2.25)$ and week $6(4.92 \pm 1.79)$ of treatment with calcipotriol was significantly reduced $(t=9.37,10.54$ and $12.43 ; \mathrm{P}<0.05,0.05$ and 0.001 , respectively).

Effect of calcipotriol on the expression levels of A20 and $N F-\kappa B$ in the skin lesions of patients with psoriasis as evaluated by immunohistochemistry. Immunohistochemical 

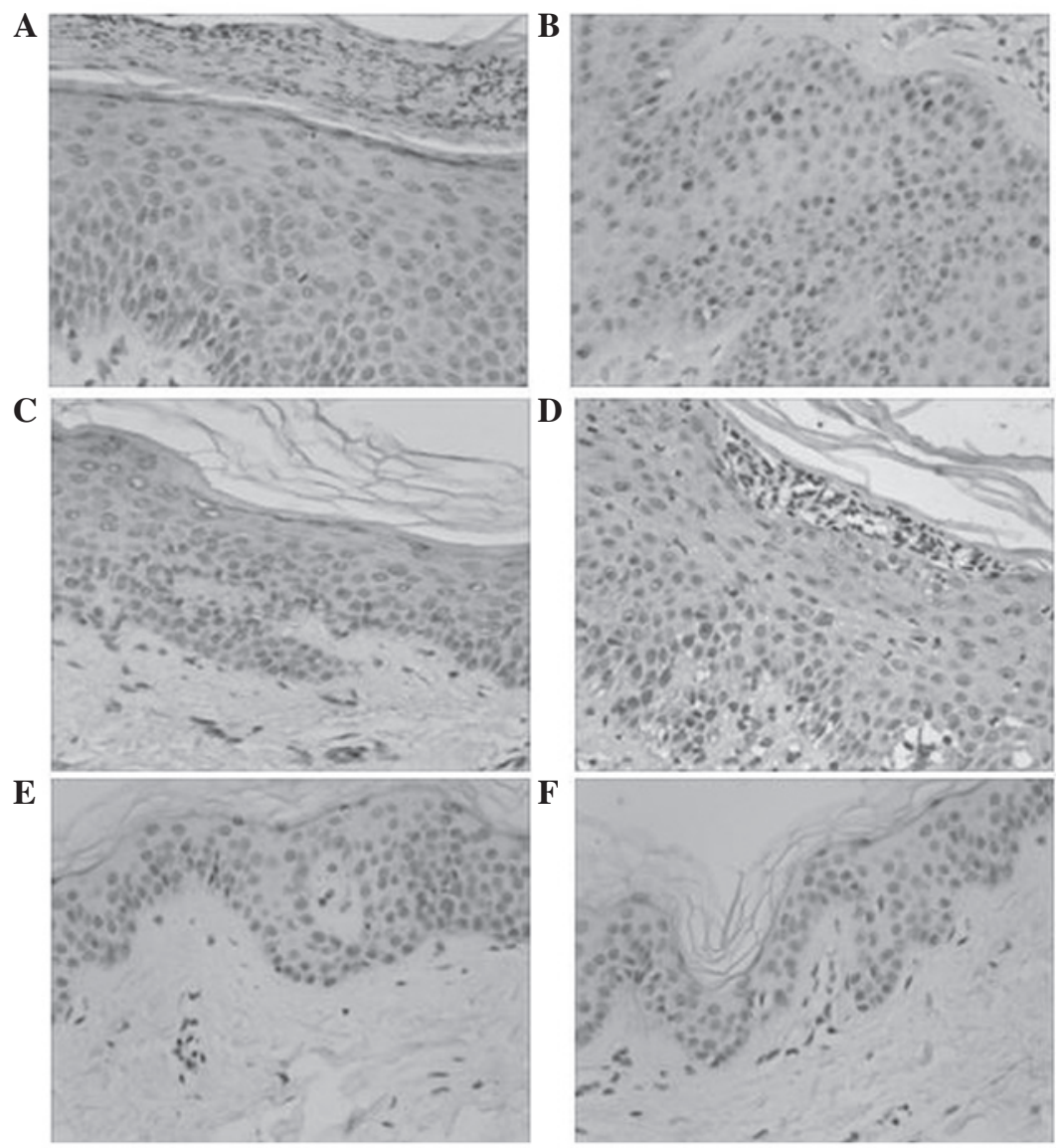

Figure 1. Expression levels of A20 and nuclear factor (NF)- $\mathrm{kB}$ in the skin lesions of patients with psoriasis (A and B) prior to and (C and D) after a 6-week treatment with calcipotriol; (E and F) reduced expression levels of A20 and NF- $\mathrm{KB}$ in normal tissue. (A, C and E) A20 was expressed in the layers of basal and prickle cells, mainly located in the membranes and cytoplasms of keratinocytes and appeared tan or dark brown in color following staining (magnification, $\mathrm{x} 200$ ). (B, D and F) NF- $\mathrm{B}$ p65 was expressed in the entire epithelial layer, mainly in the cytoplasms and nuclei of keratinocytes and appeared dark brown in color following staining (magnification, $\mathrm{x} 200$ ).

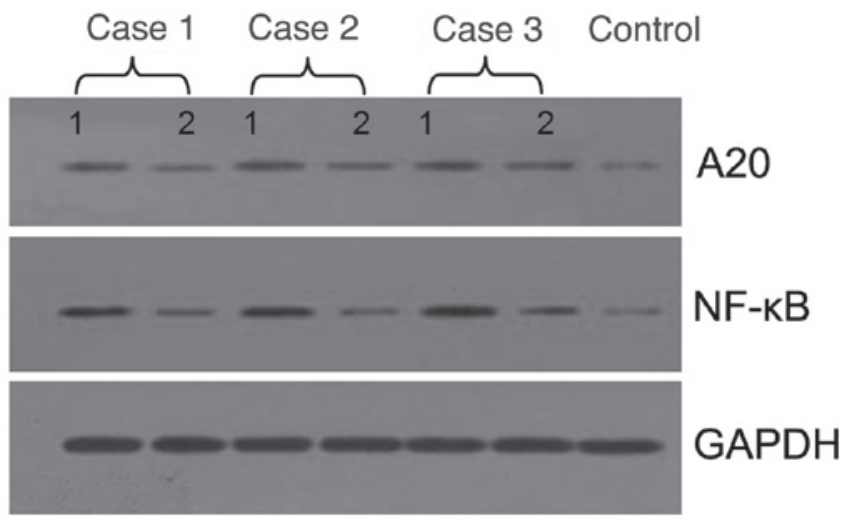

Figure 2. Expression of A20 and nuclear factor (NF)- $\kappa \mathrm{B}$ in the skin lesions of patients with psoriasis measured by western blot analysis in 3 cases prior to and at the end of calcipotriol treatment, respectively. 1, prior to treatment 2 , at the end of week 6 of calcipotriol treatment. GAPDH, glyceraldehyde 3-phosphate dehydrogenase.

staining showed that A20 was expressed in the layers of basal and prickle cells (Fig. 1), and was mainly located in the membranes and cytoplasms of keratinocytes as tan or dark brown colors. The expression levels of A20 in psoriasis skin lesions prior to treatment with calcipotriol were elevated compared with those in normal skin tissue $\left(\chi^{2}=8.34 ; \mathrm{P}<0.001\right)$.
At the end of week 6 of treatment, A20 expression levels were significantly lower in the psoriasis skin lesions compared with the same lesions prior to treatment $\left(\chi^{2}=3.65 ; \mathrm{P}<0.01\right)$. NF- $\kappa B$ p65 was expressed in the entire epithelium layer, mainly in the cytoplasms and nuclei of keratinocytes, which was exhibited as dark brown staining in the nuclei and strong tan staining in the cytoplasm. In normal skin tissue, the majority of cellular nuclei were negative for NF- $\mathrm{kB}$ p65 although the cytoplasms were partially positive $\left(\chi^{2}=9.15 ; \mathrm{P}<0.001\right)$. Following the 6 -week treatment with calcipotriol, NF- $\mathrm{KB}$ p65 expression levels were significantly decreased in the psoriasis skin lesions when compared with those in the lesions prior to treatment $\left(\chi^{2}=4.17 ; \mathrm{P}<0.01\right)$.

Effect of calcipotriol on the expression levels of A20 and $N F-\kappa B$ in the skin lesions of patients with psoriasis as evaluated by western blotting. The results of the western blot analysis indicated that the expression levels of A20 and NF- $\mathrm{KB}$ in the skin lesions of patients with psoriasis prior to treatment were significantly higher compared with those in the normal tissue (1:3.2 and 1:5.4, respectively; Fig. 2), where 1 is the intensity of the protein band in normal tissue and 3.2 and 5.4 are the intensities of the bands from the patients. However, they were significantly decreased following 6 weeks of treatment with calcipotriol (1:1.6 and 1:1.2, respectively; $\mathrm{P}<0.01)$. 


\section{Discussion}

Psoriasis is a type of immune-mediated chronic inflammatory dermatosis. It affects $1-3 \%$ of the global population and is presented pathologically as hyperkeratosis, akeratosis, hypertrophy in the prickle-cell layer and invasion of the skin by inflammatory cells (9). A previous study indicated that there is an upregulation of phosphorylated $N F-\kappa B$ in psoriasis skin lesions when compared with normal skin (1). As the key regulator in inflammation, cellular proliferation, differentiation and apoptosis, $\mathrm{NF}-\kappa \mathrm{B}$ is also considered to be the key regulator in the pathology of psoriasis; multiple types of cells, chemokines and cytokines associated with psoriasis are dependent on the activation of $\mathrm{NF}-\kappa \mathrm{B}$ signaling (10). As an example, the innate immune response protein toll-like receptor 2 (11) and caspase-5 (12) are able to recognize the upregulation of pathogen-associated molecular patterns in psoriasis and promote the fine regulation of proinflammatory cytokines through activation of the downstream signaling of $\mathrm{NF}-\kappa \mathrm{B}$.

Zinc finger protein A20 is a type of intracellular zinc finger protein that is coded by the TNFAIP3 gene. There are two $\kappa \mathrm{B}$ motifs at the -54 and -66 bps of the A20 gene, whose expression is mediated by $\mathrm{NF}-\kappa \mathrm{B}$. The basal expression level of A20 is low in the majority of cells; $\mathrm{NF}-\kappa \mathrm{B}$ is activated by multiple stimulations and is translocated into the nucleus where it binds with the $\kappa \mathrm{B}$ component of the TNFAIP3 promoter and enhances the transcription of the TNFAIP3 gene. The expressed A20 induces the destruction of the inhibitor of $\kappa \mathrm{B}$ kinase $\gamma$ (also known as the $\mathrm{NF}-\kappa \mathrm{B}$ essential modulator) to modulate the $\mathrm{NF}-\kappa \mathrm{B}$ pathway through ubiquitylation by negative feedback and also modulates TNF signaling through deubiquitylation $(13,14)$. The features of ubiquitylation and deubiquitylation allow A20 to specifically modulate the functional status of cells in order to ensure the self-preservation of organs during inflammation, thereby demonstrating dual anti-inflammatory and anti-apoptotic effects (15). Notably, genome-wide association studies found that the gene polymorphism of A20 and ABIN-1 (also known as TNFAIP-3 interacting protein 1, TNIP1) was closely associated with not only the development of psoriasis but also the efficiency of TNF inhibitors, including infliximab, adalimumab and etanercept, in the treatment of psoriasis $(16,17)$.

Since A20 has ubiquitylation and deubiquitylation functions, the present study investigated the expression changes of A20 and NF- $\kappa$ B in the skin lesions of patients with psoriasis vulgaris during treatment with calcipotriol. The results indicated that the clinical effectiveness rate was $57.69 \%(15 / 26)$, the PASI scores and the staining of A20 and NF- $\kappa$ B at the end of 6 weeks of treatment were significantly lower compared with those prior to treatment and the corresponding expression levels of proteins decreased. These results suggest that $\mathrm{A} 20$ regulates $\mathrm{NF}-\kappa \mathrm{B}$ through negative feedback and plays an important role in the treatment of psoriasis with calcipotriol. The mechanism of A20 in the regulation of apoptosis-associated factors or the signaling transduction of keratinocytes in psoriasis requires further study, which may uncover new therapeutic regimens and strategies.

\section{Acknowledgements}

This study was supported by the Youth Foundation of the Natural Science Research Project of Jiangsu Province (no. BK2012153) and the Psoriasis Vulgaris Foundation of Bright Future Pharmaceutical Laboratories Ltd, Chinese Society of Dermatology.

\section{References}

1. Lizzul PF, Aphale A, Malaviya R, Sun Y, Masud S, Dombrovskiy V and Gottlieb AB: Differential expression of phosphorylated $\mathrm{NF}-\kappa \mathrm{B} / \mathrm{RelA}$ in normal and psoriatic epidermis and downregulation of $\mathrm{NF}-\kappa \mathrm{B}$ in response to treatment with etanercept. J Invest Dermatol 124: 1275-1283, 2005.

2. Jiang X, Tian H, Fan Y, Chen J, Song Y, Wang S, Zhu F, Guo C, Zhang L and Shi Y: Expression of tumor necrosis factor $\alpha$-induced protein $3 \mathrm{mRNA}$ in peripheral blood mononuclear cells negatively correlates with disease severity in psoriasis vulgaris. Clin Vaccine Immunol 19: 1938-1942, 2012.

3. Peric M, Koglin S, Dombrowski Y, Gross K, Bradac E, Büchau A, Steinmeyer A, Zügel U, Ruzicka T and Schauber J: Vitamin D analogs differentially control antimicrobial peptide/'alarmin' expression in psoriasis. PLoS One 4: e6340, 2009.

4. van de Kerkhof PC: The psoriasis area and severity index and alternative approaches for the assessment of severity persisting areas of confusion. Br J Dermatol 137: 661-662, 1997.

5. Zhou XD, Yu JP, Liu J, Luo HS, Chen HX and Yu HG: Overexpression of cellular FLICE-inhibitory protein (FLIP) in gastric adenocarcinoma. Clin Sci (Lond) 106: 397-405, 2004.

6. Massi D, Tarantini F, Franchi A, Paglierani M, Di Serio C, Pellerito S, Leoncini G, Cirino G, Geppetti P and Santucci M: Evidence for differential expression of Notch receptors and their ligands in melanocytic nevi and cutaneous malignant melanoma. Mod Pathol 19: 246-254, 2006.

7. Wan J, Liu XM, Lei TC and Xu SZ: Effects of mutation in dopachrome tautomerase on melanosome maturation and anti-oxidative potential in cultured melanocytes. Zhonghua Yi Xue Za Zhi 89: 1707-1710, 2009 (In Chinese).

8. BandScan5.0. http://soft.bio1000.com/show-149.html. Accessed September 27, 2013.

9. Colombo GL, Di Matteo S, Bruno G, Girolomoni G and Vena GA: Calcipotriol and betamethasone dipropionate in the treatment of mild-to-moderate psoriasis: a cost-effectiveness analysis of the ointment versus gel formulation. Clinicoecon Outcomes Res 4: 261-268, 2012.

10. Tsuruta D: NF- $\kappa \mathrm{B}$ links keratinocytes and lymphocytes in the pathogenesis of psoriasis. Recent Pat Inflamm Allergy Drug Discov 3: 40-48, 2009.

11. Begon E, Michel L, Flageul B, Beaudoin I, Jean-Louis F, Bachelez H, Dubertret L and Musette P: Expression, subcellular localization and cytokinic modulation of Toll-like receptors (TLRs) in normal human keratinocytes: TLR2 up-regulation in psoriatic skin. Eur J Dermatol 17: 497-506, 2007.

12. Salskov-Iversen ML, Johansen C, Kragballe K and Iversen L: Caspase-5 expression is upregulated in lesional psoriatic skin. $\mathrm{J}$ Invest Dermatol 131: 670-676, 2011.

13. Harhaj EW and Dixit VM: Deubiquitinases in the regulation of $\mathrm{NF}-\kappa \mathrm{B}$ signaling. Cell Res 21: 22-39, 2011.

14. Vereecke L, Beyaert R and van Loo G: The ubiquitin-editing enzyme A20 (TNFAIP3) is a central regulator of immunopathology. Trends Immunol 30: 383-391, 2009.

15. Wertz IE, O'Rourke KM,Zhou H, Eby M, et al: De-ubiquitination and ubiquitin ligase domains of A20 downregulate NF- $\mathrm{B}$ signalling. Nature 430: 694-699, 2004.

16. Nair RP, Duffin KC, Helms C, Ding J, Stuart PE, Goldgar D, Gudjonsson JE, Li Y, Tejasvi T, Feng BJ, et al: Collaborative Association Study of Psoriasis: Genome-wide scan reveals association of psoriasis with IL-23 and NF- $\kappa$ B pathways. Nat Genet 41: 199-204, 2009.

17. Tejasvi T, Stuart PE, Chandran V, Voorhees JJ, Gladman DD, Rahman P, Elder JT and Nair RP: TNFAIP3 gene polymorphisms are associated with response to TNF blockade in psoriasis. J Invest Dermatol 132: 593-600, 2012. 